\title{
Profile interview with Professor Mia Crampin
}

My current role is multifaceted. I develop and maintain external partnerships and collaborations that are essential to the pursuit of strategic, high quality research

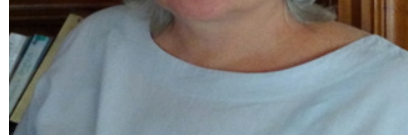

Date Received: 03 March 2019

Correspondence:

mmjeditors@medcol.mw

https://dx.doi.org/10.4314/mmj.v31i1.18

HZ: Tell me about yourself and your professional background?

MC: I grew up in Edinburgh, in Scotland and studied Medicine at Manchester University. I chose Medicine because I wanted to become a forensic pathologist; maybe I had read too many crime novels! I soon became more interested in infectious diseases, and after completing internships in infectious diseases and pathology, I was keen to work abroad in settings where infectious diseases remain important. It was during my Diploma in Tropical Medicine and Hygiene at the Liverpool School of Tropical Medicine that I became passionate about public health and epidemiology. Inspired by this experience, I went on to complete a public health clinical training scheme in southeast London, and soon after, I joined the Karonga Prevention Study (KPS) in Malawi. I arrived in Malawi in 1998 as a field epidemiologist and now I am Professor of Epidemiology at London School of Hygiene and Tropical Medicine, and Professor of Global Health Epidemiology at University of Glasgow. Just like me, KPS has changed and developed over the years and is now known as Malawi Epidemiology and Intervention Research Unit (MEIRU) and is established as an NGO; a partnership between Malawi Ministry of Health, Malawi College of Medicine, London School of Hygiene and Tropical Medicine, and University of Glasgow.

HZ: You have mentioned of your passion in infectious diseases, why did you want to work overseas? What motivated you then?

MC: In the UK, much of clinical medicine is centered on management of chronic conditions in elderly people that are associated with lifestyle; diabetes, chronic pulmonary disease, heart disease and so on. But in my early career I was fascinated by infectious disease. As a medical student in the 1980s, when the HIV epidemic was emerging, I decided to do my medical elective in HIV in New York, where they were at the forefront of HIV management. I was also interested in tropical infections, so a move to Malawi, where infectious diseases were much a bigger part of the problem, was a natural progression. And now, some twenty years later, I have come full circle, spending much of my time thinking about chronic conditions in Malawi.
HZ: Tell me a about brief history of the Karonga Prevention Study (KPS)?

MC: KPS started in 1979 with Professor Paul Fine and Dr Jorg Ponnighaus. Initially established as a study of Leprosy with support from the British Leprosy Relief Association. When I joined in 1998, we worked mostly on vaccine trials and epidemiological studies in TB and HIV with additional studies in the genetics and immunology of TB and leprosy. We established a demographic surveillance site in Karonga in 2002, which is still operating and is very highly regarded. We had a program of pneumococcal related work with Professor Neil French, and then we continued looking at the impact of the new ART programs. What become very clear by the late 2000's from our verbal autopsy work in Karonga, was that the decline in mortality rate in adults from HIV was revealing an underlying very high mortality of non-communicable diseases. In 2012 we received funding from Wellcome Trust, to establish large studies of chronic conditions. Working with the Ministry of Health we selected Area 25 in Lilongwe to establish our urban population research platform and the Community Health Sciences Unit (CHSU), for our urban headquarters. Between 2013 and 2016 we conducted a rural and urban study, recruiting 33,000 adults with collection of detailed information on NCD risk factors and health outcomes. Large epidemiological studies of this kind are necessary to understand who is the most affected in the population, what the determinants are of their conditions and later, the incidence of disease and ultimately health trajectories and survival. Particularly when you are looking at chronic conditions or even the infectious diseases which manifest over many years like Tuberculosis, HIV and so on, it is very important to be able to track people reliably over many years. We have people in Karonga whom we first saw in 1979, and we continue to be in contact with them, which enables us to do incredibly important studies on the development of disease over the life-course.

HZ: As you said, you came in as a field epidemiologist in Karonga in 1998 but now you are the Director of MEIRU, what are your responsibilities as a Director?

MC: My current role is multifaceted. I develop and maintain external partnerships and collaborations that are essential to the pursuit of strategic, high quality research. On a day-today I ensure that policy makers are informed of the work we do -whether at national, regional or international level, - by creating visibility through interfacing with our policy makers and partners. 
I am also responsible for securing substantive research grants to support the work that we do here and I also provide mentorship to our research team to ensure that they experience career development. I have the ultimate responsibility for the conduct of all studies. This includes ensuring that our studies are conducted ethically, that high quality data are collected, and that those data are turned into policy relevant findings. As Director, I also lead the senior management team, which means that I have oversight of all kind of administrative functions, in addition to the research. Fortunately I am supported by a strong team and the MEIRU trustees.

HZ: What has been the impact of MEIRU's research projects in Malawi so far and how have the findings informed clinical practice?

MC: The impact of epidemiological research studies are not always easy to identify as they tend to influence policy rather than clinical practice. For example, in the case of NCDs, we are fortunate in Malawi that the NCD Department at the Ministry of Health are very responsive. As such our findings on hypertension and diabetes have informed the Ministry's approach by highlighting that these are not urban conditions only but also significantly impacting on the rural poor. That is very important in terms of understanding how services must be delivered and made available throughout health sector in Malawi.

Having said that, we have moved rapidly from collecting observational data to intervention trials to tackle these problems. We have multiple intervention trials in progress, including a study together with the Ministry of Health and Education whereby we are developing a large cluster randomized trial of sodium reduction through a curriculum intervention in schools. It's a very scalable intervention and trials in China have shown reduced sodium intake and hypertension in whole communities using a similar approach.

HZ: What future plans and possibilities in the field of research are there for Malawi?

MC: Malawi is finding itself increasingly in a situation where people have multiple chronic conditions (for example $\mathrm{HIV}$, hypertension, diabetes, chronic lung disease, epilepsy to name a few). The extent to which these conditions are influenced by early life exposures is not well understood but early life influences are likely to be important. Research in this area will help us to understand better how to manage and ideally to mitigate the consequences.

There are a combination of factors like poor nutrition in pregnancy and infancy, repeated exposure to infectious diseases, environmental exposures and stress that build up a burden of chronic conditions. At present we are planning a large chronic conditions cohort that will, pending funding support, explore all of these issues so that we can understand the origins of these diseases at very early life and how to prevent them.

HZ: As someone who has worked with MEIRU for quite a very long time, are there challenges you have met in your work?

MC: As a small research organization, one of our main challenges is that we don't have the benefit of core funding that large institutions receive. This means that we need to work very hard to secure grants to sustain not only our research projects but also our research infrastructure. As small can be beautiful, there is a good argument for being modestly sized and focusing on excellence rather than growth.

Supporting young Malawian researchers throughout their careers is also challenging but we are proud that many of our staff go on to develop successful careers in other good institutions. Malawi has a growing cadre of impressive researchers and ideally in 10 to 20 years' time, senior Malawian researchers will lead all of the research affiliates. We were lucky enough to have Professor. Moffat Nyirenda as our director for few years before he took up his post in Uganda.

HZ: You are also the leader of African Noncommunicable Disease Longitudinal data Alliance (ANDLA), what is it like to lead this consortium?

MC: I can honestly say ANDLA is a partnership rather than an organization with a leader. I applied for the money to establish it, but it is a network of seven different partner institutions in Africa. One in Kenya, one in Uganda, one in Malawi, two in Tanzania, two in South Africa, all very well-known institutions. We harmonize longitudinal data on chronic conditions and at the moment we are looking at cardiovascular risk scores, and how to validate them for African populations. Most of us have been working together for a long time on HIV data so we know each other very well and have the trust to share datasets.

HZ: What outstanding life experiences or achievements are you proudest of?

MC: I am very proud of MEIRU and our approach to longitudinal studies and I think it does give a sense of pride when that's recognized as one of most rigorously collected and best datasets for answering particular questions from the region. That takes a lot of persistence!

HZ: You have spent quite a lot of time in Malawi, from 1998 up to date, is there something in particular that you like in Malawi.

MC: It's no secret that I loved living in Chilumba, and for me it was the perfect place to raise a family. My daughters grew up running around outside with their friends and had lot of freedom. Maybe because I am Scottish I felt very at home between the mountains and the lake, and when I moved from London to Malawi I felt I had come home because of the friendliness I also recognized from growing up in Scotland.

HZ: Thank you so much for your time and insights into your professional and personal life. Do you have any last words?

MC: In addressing chronic conditions it is going to be very important to have multi-sectoral actions; involving agricultural, involving town-planning and involving industry to improve people's health and wellbeing. Health researchers will need to become much more expert in fostering these collaborations for the benefit of public health. That is the challenge for MEIRU over the next few years. 